\title{
The Italian version of rheumatoid arthritis pain scale (IT-RAPS): psychometric properties on community and clinical samples
}

\begin{abstract}
M. Tofani ${ }^{1,2}$, M. Nobilia ${ }^{2,3}$, G. Culicchia ${ }^{2,3}$, G. Esposito ${ }^{2,4}$, A. Savona ${ }^{4}$, I. Tashi ${ }^{4}$, A. Ventura ${ }^{5}$, G. Galeoto ${ }^{2,6}$

${ }^{1}$ Ospedale Pediatrico Bambin Gesù, Dipartimento di Neuroscienze e Neuroriabilitazione, Palidoro (RM), Italy;

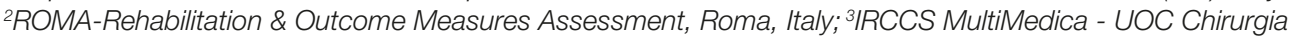
della mano, Milano, Italy; ${ }^{4}$ Sapienza-Università di Roma, Dipartimento di Scienze Anatomiche, Istologiche, Forensi e Ortopediche, Roma, Italy; IITOP SpA Officine Ortopediche, Palestrina (RM), Italy;

${ }^{6}$ Department of Public Health and Infectious Diseases
\end{abstract}

\section{SUMMARY}

This paper describes the validation process of the Italian version of the Rheumatoid Arthritis Pain Scale (ITRAPS), describing its translation and adaptation to Italian culture.

The cultural adaptation and validation were based on data from a sample of people affected by rheumatoid arthritis (RA). The process required a forward and backward translation of the original language, reviewed by an expert panel. The adapted version of the RAPS was then tested on a community and clinical sample, in order to test its psychometric properties.

The IT-RAPS was submitted to 122 people affected by RA. The data was analyzed using Cronbach's coefficient alpha and the Pearson product-moment correlation coefficients. The IT-RAPS showed an internal consistency reliability coefficient of 0.96 .

This is the first study reporting the validation and cross-cultural adaptation of the RAPS in Italian. The study's findings provided support for the IT-RAPS as a reliable and valid measurement of multidimensional pain in RA patients.

Key words: Rheumatoid arthritis; Pain; Outcome measure.

Reumatismo, 2019; 71 (1): 13-18

\section{INTRODUCTION}

Tn Italy, there are approximately 280.000 - people who have a diagnosis of rheumatoid arthritis (RA) (1). The prevalence of active $R A$, remission $R A$ and confirmed $R A$ (active + remission RA) is 0.32 percent, 0.09 percent and 0.41 percent $(95$ percent CI 0.38-0.44), respectively (2).

Patients' perceptions regarding treatment goals, specifically those of preventing disability, pain and psychological symptoms, can differ from those of clinicians (3). Pain is the area of health in which almost 70 percent of RA patients would like to see improvement (4).

In the clinical literature there are many scales which assess pain, such as the McGill Pain Questionnaire (5), which measures the dif- ferent qualities of the subjective pain experience, as well as the Visual Analogue Scale and the Verbal Descriptor Scale (6), which assess pain severity. These assessment tools refer only to the body function component of the World Health Organization (WHO) International Classification of Functioning, Disability and Health (ICF) (7).

Turk et al. (8) describe a biopsychosocial model of chronic pain which highlights the importance of people's appraisals of their symptoms, their ability to self-manage pain and related problems, and their fears about pain and injury that motivate efforts to avoid exacerbation of symptoms and further injury or re-injury. It seems necessary to use a holistic approach to assess pain in RA patients. We chose the RAPS for the following reasons: its simplicity and its speed of admin-
Corresponding author: Marco Tofani

Dipartimento di Neuroscienze e Neuroriabilitazione, Ospedale Pediatrico Bambin Gesù Via della Torre di Palidoro snc 00050 Palidoro (RM), Italia E-mail: marco.tofani@opbg.net 
istration, and because it refers to the body function, body structure, impairment and activity limitations in the WHO ICF components (7). Based on gate control and affective motivational theories, the RAPS (9) is composed of theoretical subscales that represent indicators of the total pain experience that are unique to RA. The subscales include physiological, affective, sensorydiscriminative and cognitive factors. The 24 items on the self-administered scale were scored using a seven-point Likert scale ranging from never to always.

A MEDLINE search was performed: only the Indian validation study was found. The Indian version of the RAPS (10) was found to be a valid and clinically relevant instrument for measuring pain in Indian patients suffering from RA.

The purpose of this study is the translation, cultural adaptation and validation of the RAPS in a group of RA individuals with clinical evidence of pain, in order to define psychometric properties in an Italian sample.

\section{MATERIALS AND METHODS}

The original English version of the RAPS has no copyright; it was translated into Italian and was culturally adapted according to the census bureau guidelines for the translation of a data collection instrument (11). The assessment tool was forward translated into Italian by two health professionals (an occupational therapist and a physical therapist). Then, a bilingual expert panel (a rheumatologist and a physiatrist) identified and resolved inappropriate translations and compared the forward-translated version of the RAPS with the original; the result was a preliminary draft of the scale in the target language. Afterwards, an independent translator whose mother tongue is English back-translated the document. The backtranslated version was compared with the original. Finally, in order to optimize the cultural adaptation, the involved experts synthesized the results into a pre-final Italian version of the RAPS (IT-RAPS).

The validation process was based on data from both women and men, age 18 years or older, who had experienced pain of at least three months' duration. The sample was recruited from the outpatient clinic of Sapienza Università di Roma and from a community setting, thanks to collaboration with an organization of rheumatic patients of the Lazio Region (ALMAR - Associazione Laziale Malati Reumatici). All of the subjects had to have a minimum level of primary education, had to be able to understand instructions for answering all of the questions and had to sign an informed consent document (12).

In the pretesting process, we wanted to determine whether any differences were present in the administration of the tests between the version in the literal translation and those which were culturally adapted. According to Perneger et al., small samples taken from the usual participants in pretest questionnaires may fail to uncover even the most common problems (false negatives). A default sample size of 30 participants is recommended (13).

The internal consistency was used to assess the homogeneity of the scale and the interrelatedness of the items; the Cronbach's $\alpha$ coefficient should be at least 0.70 .

The intraclass correlation coefficient (ICC) was used to assess test-retest reliability. To evaluate the intra-rater reliability, the same patients were evaluated twice over the course of seven days. The scale was considered as stable at the test-retest for ICC $>0.70$ (14).

The concurrent validity was evaluated by comparing the IT-RAPS with the Visual Analogue Scale (VAS) (6). The comparison with the gold standard was examined using Pearson's method.

The sampling adequacy was detected through the Kaiser-Meyer-Olkin test (KMO) and Bartlett's test of sphericity. The factorial structure test was determined through the analysis of the main components with oblique rotation with the maximum likelihood solution. This was done in agreement with the recommendations of Graetz, who argues that with oblique rotation, we get more appropriate results and can provide a simple solution that is easy to interpret. The acceptable factor loading score was set $\geq 0.4$. 


\section{RESULTS}

\section{Translation and cross-cultural adaptation}

To avoid bias due to translation, 29 subjects were tested randomly. They reported

Table I - Sample characteristics.

\begin{tabular}{|l|c|}
\hline Age $($ mean $\pm S D)$ & $58 \pm 12$ \\
Gender $n(\%)$ & $98(80.4)$ \\
Women & $24(19.6)$ \\
Men & \\
\hline Pain duration $n(\%)$ & $58(47.6)$ \\
3-6 months & $34(27.8)$ \\
7-10 months & $30(24.6)$ \\
More than 11 months & \\
\hline Education $n(\%)$ & $26(21.3)$ \\
Secondary School & $83(68.1)$ \\
High School & $13(10.6)$ \\
University & \\
\hline
\end{tabular}

semantic incongruities and suggested enhancing solutions for the cross-cultural adaptation. From the analysis of the report, it was possible to draw up the final version of the IT-RAPS.

\section{Sample characteristics}

For the study, 122 subjects with a diagnosis of RA were included ( 80.4 percent females; 19.6 percent males). The mean age was $58 \pm 12$ years (range $27-79$ ). The characteristics of the sample are summarized in Table I.

\section{Internal consistency}

The Cronbach's coefficient alpha, assessing the internal consistency for the total of IT-RAPS, was found to be 0.96 . This value exceeds the minimum for a new instrument

Table II - Cronbach's coefficient alpha item-total correlation.

\begin{tabular}{|l|c|c|c|c|}
\hline & Scale mean if item deleted & Scale variance item deleted & Corrected item-total correlation & Cronbach's alpha if item deleted \\
\hline Q1 & 71.50 & 1205.622 & 0.722 & 0.963 \\
Q3 & 71.40 & 1199.342 & 0.805 & 0.963 \\
Q4 & 71.47 & 1201.747 & 0.728 & 0.964 \\
Q5 & 70.91 & 1205.469 & 0.763 & 0.963 \\
Q6 & 71.42 & 1202.588 & 0.761 & 0.963 \\
Q7 & 71.33 & 1219.701 & 0.690 & 0.964 \\
Q8 & 70.95 & 1238.646 & 0.507 & 0.966 \\
Q9 & 71.87 & 1200.964 & 0.791 & 0.963 \\
Q10 & 71.20 & 1203.565 & 0.777 & 0.963 \\
Q11 & 71.00 & 1193.640 & 0.802 & 0.963 \\
Q12 & 71.77 & 1212.666 & 0.666 & 0.964 \\
Q13 & 71.13 & 1204.243 & 0.698 & 0.964 \\
Q14 & 71.77 & 1222.630 & 0.618 & 0.965 \\
Q15 & 71.86 & 1215.457 & 0.615 & 0.965 \\
Q16 & 71.29 & 1199.615 & 0.758 & 0.963 \\
Q17 & 72.04 & 1205.999 & 0.719 & 0.964 \\
Q18 & 71.17 & 1204.088 & 0.783 & 0.963 \\
Q19 & 71.28 & 1196.544 & 0.840 & 0.963 \\
Q20 & 71.85 & 1218.238 & 0.629 & 0.965 \\
Q21 & 71.13 & 1206.993 & 0.724 & 0.964 \\
Q22 & 71.51 & 1207.171 & 0.716 & 0.964 \\
Q23 & 71.85 & 1230.220 & 0.587 & 0.965 \\
Q24 & 71.57 & 1199.725 & 0.793 & 0.963 \\
\hline & 71.07 & 1203.671 & 0.748 & \\
\hline
\end{tabular}


Table III - Inter-rater test-retest reliability.

\begin{tabular}{|c|c|c|c|}
\hline Item & Test & Retest & ICC (IC95\%) \\
\hline Q1 & $2.37 \pm 2.18$ & $2.83 \pm 2.25$ & $0.817(0.647-0.910)$ \\
\hline Q2 & $2.57 \pm 2.19$ & $2.93 \pm 2.2$ & $0.926(0.849-0.965)$ \\
\hline Q3 & $2.33 \pm 2.18$ & $2.38 \pm 2$ & $0.754(0.540-0.893)$ \\
\hline Q4 & $3.37 \pm 2.25$ & $3.38 \pm 1.8$ & $0.785(0.592-0.893)$ \\
\hline Q5 & $2.6 \pm 3.37$ & $2.86 \pm 1.8$ & $0.876(0.753-0.940)$ \\
\hline Q6 & $2.8 \pm 2.1$ & $2.59 \pm 1.8$ & $0.853(0.710-0.928)$ \\
\hline Q7 & $3.2 \pm 2.3$ & $2.86 \pm 2.3$ & $0.790(0.600-0.896)$ \\
\hline Q8 & $2.23 \pm 2$ & $2.55 \pm 2$ & $0.771(0.568-0.886)$ \\
\hline Q9 & $2.87 \pm 2.19$ & $3.17 \pm 2.1$ & $0.934(0.865-0.969)$ \\
\hline Q10 & $2.57 \pm 2.23$ & $2.72 \pm 2$ & $0.751(0.647-0.910)$ \\
\hline Q11 & $1.93 \pm 2.21$ & $2.24 \pm 2.1$ & $0.787(0.596-0.894)$ \\
\hline Q12 & $3.07 \pm 2.2$ & $2.66 \pm 2.1$ & $0.810(0.647-0.911)$ \\
\hline Q13 & $2.2 \pm 2.1$ & $2.14 \pm 2.2$ & $0.895(0.789-0.949)$ \\
\hline Q14 & $1.9 \pm 2.3$ & $2.34 \pm 2.3$ & $0.830(0.670-0.917)$ \\
\hline Q15 & $2.33 \pm 2.1$ & $2.97 \pm 2.16$ & $0.702(0.546-0.856)$ \\
\hline Q16 & $1.63 \pm 2$ & $1.62 \pm 1.97$ & $0.884(0.768-0.944)$ \\
\hline Q17 & $2.8 \pm 2.1$ & $2.66 \pm 2.1$ & $0.764(0.556-0.882)$ \\
\hline Q18 & $2.63 \pm 2.3$ & $2.97 \pm 2.16$ & $0.848(0.702-0.926)$ \\
\hline Q19 & $2.27 \pm 2.1$ & $2.79 \pm 2.1$ & $0.733(0.517-0.894)$ \\
\hline Q20 & $2.9 \pm 2.2$ & $2.62 \pm 2$ & $0.903(0.803-0.953)$ \\
\hline Q21 & $2.37 \pm 2.4$ & $2.62 \pm 2$ & $0.810(0.634-0.906)$ \\
\hline Q22 & $2.53 \pm 2.17$ & $2.1 \pm 2$ & $0.748(0.530-0.873)$ \\
\hline Q23 & $2.1 \pm 2.13$ & $2.3 \pm 1.9$ & $0.907(0.812-0.955)$ \\
\hline Q24 & $2.57 \pm 2.2$ & $2.24 \pm 2$ & $0.839(0.686-0.921)$ \\
\hline Total & $60.27 \pm 42.96$ & $62.18 \pm 41$ & $0.943(0.880-0.973)$ \\
\hline & & & \\
\hline & & & \\
\hline
\end{tabular}

and is a strong indicator of internal consistency. Correlations of each item with the IT-RAPS were all positive and significant $(p<0.01)$ with correlations between 0.4 and 0.8. The evaluation of Cronbach's coefficient alpha of the sub-scales indicated significant correlations: sensory-discriminative alpha $=0.92$, affective component $=0.79$, physiologic component $=0.84$ and cognitive component $=0.89$ (Table II).

Test-retest reliability showed statistically significant data, with ICC $>0.7$ and $\mathrm{p}<0.01$ (Table III).

\section{Concurrent validity}

Using the Pearson correlations coefficient, the correlation between IT-RAPS and VAS was calculated. The correlations between VAS and total IT-RAPS was 0.77 with a P value $<0.01$. Correlation between VAS and IT-RAPS sub-scales are summarized in Table IV.

\section{Factor analysis}

Factor analysis with oblique rotation with the maximum likelihood solution was used to estimate construct validity of the ITRAPS. Factor analysis extracted four subscales explaining $70.34 \%$ of the variance of the test. Factor 1 includes seven items (Q1$5, \mathrm{Q} 12, \mathrm{Q} 18)$ that explain $56.28 \%$ of the variance. Factor 2 includes ten items $(\mathrm{Q} 8$, Q11, Q13, Q16, Q19-24) that explain 5.2\% of the variance. Factor 3 includes four items (Q10, Q14, Q15, Q17) that explain 4.8\% of the variance. Factor 4 includes three items (Q6, Q7, Q9) that explain 3.9\% of the variance. The values of the factor analysis are summarized in Table V.

\section{DISCUSSION AND CONCLUSIONS}

This study aimed to translate and culturally adapt the RAPS for the Italian population as well as to evaluate its psychometric properties in RA patients.

Following international guidelines, the process of translation and cultural adaptation was performed and a final version with 24 items of the IT-RAPS has resulted.

The evaluation of the internal consistency through the Cronbach's alpha was 0.96 comparable with the value of the original version (0.92) and those of Indian validation (0.91). A Cronbach coefficient alpha

Table IV - Pearson correlation between IT-RAPS subscales and VAS.

\begin{tabular}{|c|c|c|c|c|c|}
\hline & $\begin{array}{c}\text { Physiological } \\
\text { component }\end{array}$ & $\begin{array}{c}\text { Affective } \\
\text { component }\end{array}$ & $\begin{array}{c}\text { Cognitive } \\
\text { component }\end{array}$ & $\begin{array}{c}\text { Sensory-discriminative } \\
\text { component }\end{array}$ & IT-RAPS total \\
\hline VAS & $0.70^{\star}$ & $0.75^{*}$ & $0.77^{*}$ & $0.72^{*}$ & $0.77^{*}$ \\
\hline
\end{tabular}

${ }^{*}$ Correlation is significant at the 0.05 level (2-tailed). 
Table V - IT-RAPS factor analysis.

\begin{tabular}{|c|c|c|c|c|}
\hline \multirow{2}{*}{ Questions } & \multicolumn{4}{|c|}{ Component } \\
\hline & 1 & 2 & 3 & 4 \\
\hline Q1 - I would describe my pain as gnawing & 0.775 & & & \\
\hline Q2 - I would describe my pain as aching & 0.791 & & & \\
\hline Q3 - I would use the word exhausting to describe my pain & 0.741 & & & \\
\hline Q4 - I would describe my pain as annoying & 0.594 & & & \\
\hline Q5 - I am constantly in pain & 0.705 & & & \\
\hline Q6 - I would describe my pain as rhythmic & & & & 0.521 \\
\hline Q7 - I have swelling of the least one joint & & & & 0.778 \\
\hline Q8 - I have morning stiffness of one hour or more & & 0.476 & & \\
\hline Q9 - I have pain on motion of at least one joint & & & & 0.668 \\
\hline Q10 - I cannot perform all the everyday tasks I normally would because of pain & & ( & 0.675 & \\
\hline Q11 - Pain interferes with my sleep & 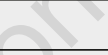 & 0.701 & & \\
\hline Q12 - I cannot decrease my pain by using methods other than taking extra medication & 0.490 & & & \\
\hline Q13 - I would describe my pain as burning & & 0.468 & & \\
\hline Q14 - I find that I guard my joints to reduce pain & & & 0.737 & \\
\hline Q15 - I brace myself because of the pain & & & 0.568 & \\
\hline Q16 - My pain is throbbing in nature & & 0.621 & & \\
\hline Q17 - I would describe my pain as sharp & & & 0.569 & \\
\hline Q18 - I would say my pain is severe & 0.578 & & & \\
\hline Q19 - I feel stiffness in my joints after rest & & 0.737 & & \\
\hline Q20 - My joints feel hot & & 0.585 & & \\
\hline Q21 - I feel anxious because of pain & & 0.464 & & \\
\hline Q22 - I would describe my pain as tingling & & 0.482 & & \\
\hline Q23 - I feel my pain is uncontrollable & & 0.624 & & \\
\hline Q24 - I feel helpless to control my pain & & 0.717 & & \\
\hline
\end{tabular}

value $>0.70$ demonstrates a good internal consistency of the scale. The study also evaluated the item-item and item-total correlation, which showed statistically significant data. The assessment through the ICC showed all values greater than 0.70 ; this finding demonstrates a good internal consistency of the IT-RAPS.

The concurrent validity showed positive and statistically significant correlations between the IT-RAPS and the VAS, and between IT-RAPS subscales and VAS. This correlation is comparable to the value obtained in the original validation, and greater than the Indian validation.

Factor analysis suggests that the IT-RAPS has four subscales such as the validation in the original language. In contrast with the finding of the original author, our factor analysis showed four factors with all values $\geq 0.40$. It seems possible to hypothesize that our factors correspond with the four subscales of IT-RAPS: physiologic, affective, cognitive and sensory-discriminative. This aspect is probably due to cross-cultural adaptation performed by involving patients' organization; their contributions led to a better comprehension of the item according to cultural and social aspects of the target population.

Further research to understand the relationship between pain perception and disease duration with a bigger sample size is required. Based on our finding, the IT-RAPS shows good psychometric properties and it could be used by physicians and health 
professionals to evaluate multidimensional pain in RA patients.

Conflict of interest: the authors declare no conflict of interest.

Acknowledgments: the above research is the result of a study project in collaboration with non-profit organizations. We thank ALMAR - Associazione Laziale Malati Reumatici for the social commitment and professionalism with which it conducts its work in favor of RA patients. We also thank ROMA - Rehabilitation and Outcome Measures Assessment for the passion and enthusiasm with which it spreads evidence of best practice in the field of rehabilitation. Lastly, we express our appreciation to all patients who cooperated with us in carrying out this research project.

\section{REFERENCES}

1. Mennini FS, Marcellusi A, Gitto L, Iannone F. Economic burden of rheumatoid arthritis in Italy: possible consequences on anti-citrullinated protein antibody-positive patients. Clin Drug Investig. 2017; 37: 375-86.

2. Rossini M, Rossi E, Bernardi D, et al. Prevalence and incidence of rheumatoid arthritis in Italy. Rheumatol Int. 2014; 5: 659-64.

3. Suarez-Almazor ME, Conner-Spady B. Rating of arthritis health states by patients, physicians, and the general public. Implications for costutility analyses. J Rheumatol. 2001; 28: 648-56.

4. Heiberg T, Tore KK. Preferences for improved health examined in 1,024 patients with rheumatoid arthritis: pain has highest priority. Arthritis Care Res. 2002; 47: 391-7.
5. Melzack R. The McGill Pain Questionnaire: major properties and scoring methods. Pain. 1975; 1: 277-99.

6. Keefe KD. The pain chart. Lancet. 1948; 2: 6-8.

7. Burckhardt CS, Kim DJ. Adult measures of pain: The McGill Pain Questionnaire (MPQ), Rheumatoid Arthritis Pain Scale (RAPS), Short-Form McGill Pain Questionnaire (SFMPQ), Verbal Descriptive Scale (VDS), Visual Analog Scale (VAS), and West Haven-Yale Multidisciplinary Pain Inventory (WHYMPI). Arthritis Care Res. 2003; S5: 96-104.

8. Turk DC, Akiko O. Psychological factors in chronic pain: evolution and revolution. J Consult Clin Psychol. 2002; 70: 678.

9. Anderson DL. Development of an instrument to measure pain in rheumatoid arthritis: Rheumatoid Arthritis Pain Scale (RAPS). Arthritis Care Res. 2001; 45: 317-23.

10. Kianifard T, Kianyfard T, Chopra A. Validation and relevance of Rheumatoid Arthritis Pain Scale (RAPS) in Indian (Asian) patients suffering from rheumatoid arthritis. Clin Rheumatol. 2016; 35: 63-71.

11. Wild D, Grove A, Martin M, et al. Principles of good practice for the translation and cultural adaptation process for patient-reported outcomes (PRO) measures: report of the ISPOR Task Force for Translation and Cultural Adaptation. Value Health. 2005; 8: 94-104.

12. Galeoto G, De Santis R, Marcolini A, et al. Il consenso informato in terapia occupazionale: proposta di una modulistica. G Ital Med Lav Ergon. 2016; 38 :107-15.

13. Perneger TV, Courvoisier DS, Hudelson PM, Gayet-Ageron A. Sample size for pretests of questionnaires. Qual Life Res. 2015; 24: 147-51.

14. Hallgren KA, Computing inter-rater reliability for observational data: an overview and tutorial. Tutor Quant Methods Psycho. 2012; 8: 23-34. 